\title{
THE RELATIONSHIP BETWEEN THE STOMATOGNATHIC SYSTEM AND BODY POSTURE
}

\author{
Antonino Cuccia, Carola Caradonna
}

doi: 10.1590/S1807-59322009000100011

Cuccia A, Caradonna C. The relationship between the stomatognathic system and body posture. Clinics. 2009;64(1):61-6.

In recent years, many researchers have investigated the various factors that can influence body posture: mood states, anxiety, head and neck positions, oral functions (respiration, swallowing), oculomotor and visual systems, and the inner ear. Recent studies indicate a role for trigeminal afferents on body posture, but this has not yet been demonstrated conclusively. The present study aims to review the papers that have shown a relationship between the stomatognathic system and body posture. These studies suggest that tension in the stomatognathic system can contribute to impaired neural control of posture. Numerous anatomical connections between the stomatognathic system's proprioceptive inputs and nervous structures are implicated in posture (cerebellum, vestibular and oculomotor nuclei, superior colliculus). If the proprioceptive information of the stomatognathic system is inaccurate, then head control and body position may be affected. In addition, the present review discusses the role the myofascial system plays in posture. If confirmed by further research, these considerations can improve our understanding and treatment of muscular-skeletal disorders that are associated with temporomandibular joint disorders, occlusal changes, and tooth loss.

KEYWORDS: Body posture; Temporomandibular joint; Temporomandibular disorders; Trigeminal system; Fascial system.

\section{INTRODUCTION}

Posture refers to the position of the human body and its orientation in space. Posture involves muscle activation that, controlled by the central nervous system (CNS), leads to postural adjustments ${ }^{1}$. Postural adjustments are the result of a complex system of mechanisms that are controlled by multisensory inputs (visual, vestibular, and somatosensory) integrated in the CNS.

Through mechanisms of feed-back and feed-forward, postural adjustments play a critical role in orthostatic and dynamic postural control, influencing the ability to perform daily living activities. As with reflexes, postural adjustments improve through exercise and learning. ${ }^{1}$ These adjustments are evoked by several types of afferent inputs: exteroceptive (skin sensitiveness of feet), proprioceptive (especially from the cervical, hip, ankle, and knee joints), vestibular (utriculus, sacculus, semicircular canals), and

Department of Oral Sciences, University of Palermo - Palermo/Italy. Email: cucciaam@odonto.unipa.it Tel.: 0039-091-6811287

Received for publication on July 22, 2008

Accepted for publication on September 03, 2008 visual (movement of the surrounding environment). These afferent inputs can be modulated by many factors, such as mood state and anxiety.

The stomatognathic system (SS) also plays an important role in postural control. The $\mathrm{SS}$ is a functional unit characterized by several structures: skeletal components (maxilla and mandible), dental arches, soft tissues (salivary glands, nervous and vascular supplies), and the temporomandibular joint and masticatory muscles (MM). These structures act in harmony to perform different functional tasks (to speak, to break food down into small pieces, and to swallow). ${ }^{3}$ In particular, the temporomandibular joint makes muscular and ligamentary connections to the cervical region, forming a functional complex called the "craniocervico-mandibular system." The extensive afferent and efferent innervations of the SS are reflected in the extensive representation of the orofacial district in the motor and sensory areas of the cerebral cortex. ${ }^{4}$

The main disorders of the cranio-cervico-mandibular system, which often affect human posture, are the temporomandibular disorders (TMD). These are a group of diseases affecting the MM, temporomandibular joint, and surrounding structures. 
Patients with TMD report bilateral or unilateral pain as their primary symptom, typically triggered by jaw movements or by palpation, where the pain sometimes radiates to the temples or to the neck. Although an increasing amount of information is available on the effects of SS on the postural body, ${ }^{4-8}$ its mechanisms remain unclear.

The aim of this literature review is to increase awareness of the fact that a postural disorder is an alteration with both medical and dental implications. A secondary goal is to promote an interdisciplinary approach to treating patients with postural disorder.

\section{LITERATURE SEARCH STRATEGY AND INCLU- SION CRITERIA}

A search of the databases Medline, PubMed, Embase, Web of Sciences, Cochrane Library, Cinahl, and Health Star was conducted for all publications related to the topic, with no limitations of language or time period. Relevant information was also derived from reference lists of the publications retrieved. The key words used in the search were body posture, temporomandibular joint, temporomandibular disorders, masticatory muscles, craniofacial pain, malocclusion, trigeminal system, and fascial system.

\section{STOMATOGNATHIC SYSTEMAND BODY POSTURE}

Evidence continues to accumulate that untreated diseases of SS, in particular temporomandibular disorders and malocclusion, carry a risk of the development of postural disorders. Recent studies emphasize the potential role of dental occlusion and of trigeminal afferents in maintaining postural control. ${ }^{6-10}$ Several studies indicate that different mandibular positions induce variations in body posture. For example, a change in the mandibular position, which can lead to changes in the proprioceptive and periodontal afferents, may affect the center of foot pressure (COP) position and gait stability. ${ }^{11,12}$ Conversely, changes in body posture may affect mandibular position. ${ }_{13,1}$

An influence of periodontal receptors on body posture is hypothesized by Gangloff and Perrin (2002), who found a significant alteration of postural control after unilateral truncular anesthesia of the mandibular nerve. ${ }^{15}$ In fact, unilateral anesthesia of the trigeminous causes a shift in body weight onto the contralateral limb, which leads the homolateral inferior limb to contract. A more symmetric mandibular position also results in a more symmetric contraction pattern of the sternocleidomastoid muscle (SCM) and it reduces body sway. ${ }^{16}$ Other studies further suggest that dental occlusion may influence body posture and spine curvature (e.g., scoliosis and lordosis).
Positive correlations between different craniofacial morphologies and postural attitudes have been reported, such as an anteriorly displaced posture in class II malocclusion, and a posteriorly displaced posture in class III malocclusion. ${ }^{17}$ Lippold et al. (2006) have shown correlations between craniofacial parameters and back shape profiles: patients with distal and vertical craniofacial patterns present higher than normal upper thoracic, lumbarlordotic, and pelvic angles (Figure 1). ${ }^{18}$ Furthermore, patients with idiopathic scoliosis show higher frequencies of malocclusion than control patients; these include Angle Class II malocclusions, lateral crossbites, lower midline deviations, and facial asymmetries. ${ }^{19-21}$

When the occlusal relationship is lost either unilaterally or bilaterally, the body posture may take on an unusual position, causing neck or shoulder pain. In these cases, a dental splint restores a balanced occlusion and determines a more symmetric maxillomandibular position and muscular activity, thereby reducing the range of body oscillation. ${ }^{22}$ In edentulous subjects, the reconstruction of occlusal support improves reaction time, based on an index of physical quickness. ${ }^{23}$ In females with deep-bite, isometric strength of the cervical flexors and deltoids varied significantly, which affected the vertical dimension of occlusion. ${ }^{24}$

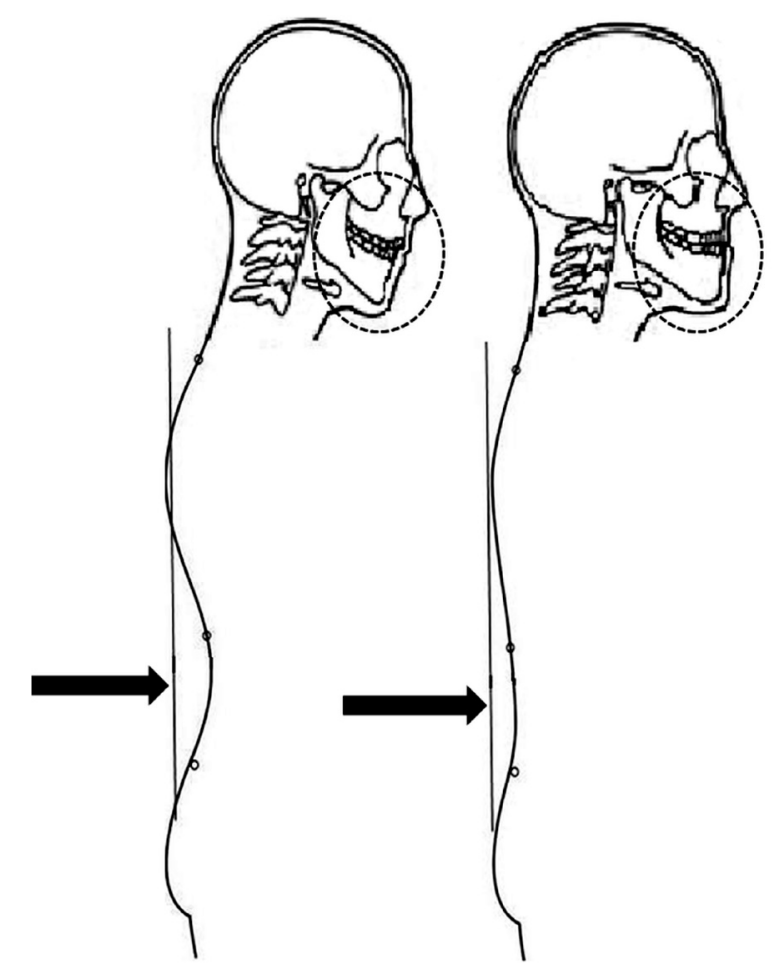

Figure 1 - Correlation between craniofacil parameters and back shape profiles: patients with distal and vertical craniofacial patterns present higher than normal upper thoracic, lumbar-lordotic, and pelvic angles; patients with mesial and horizontal craniofacial pattern present smaller than normal upper thoracic, lumbar-lordotic, and pelvic angles. Figure modified from Lippold et al. (1971) 
Studies have also demonstrated a relationship between TMDs and posture. Patients with TMD show greater changes in the body's center of gravity. ${ }^{25}$ Several studies have shown that patients with TMD present an excessively forward head position, ${ }^{26,27}$ usually associated with the shortening of the posterior cervical extensor muscles (suboccipital, semispinalis, splenii, and upper trapezius muscles), as well as shortening of the SCM. ${ }^{28}$ Anterior displacement of the head lowers the field of vision and, in the attempt to improve the field of vision, cervical lordosis increases. ${ }^{29,30}$ An anterior position of the head also influences the position of the center of gravity, which confirms the relationship between body posture and TMD. ${ }^{31-34}$ In the same way, the postural changes of the cervical region can cause TMD, modifying the orientation of the head and consequently the mandibular position. ${ }^{35}$ Previous investigations using posturography have described a correlation between body posture and gaze balancing in subjects whose trigeminal afferents were suppressed by unilateral anesthesia. ${ }^{15,36}$

All of the above studies suggest that portions of the trigeminal system strongly influence the coordination of posture and sight. However, not all studies in the literature support a relationship between posture and SS. Ferrario et al. (1996) did not observe modification of foot COP in healthy women, women with asymmetric malocclusion or different dental positions, or women with temporomandibular disorders. ${ }^{37}$ In another study, Ferrario et al. (2001) demonstrated that the muscular performances of the biceps brachii muscle were not influenced by a morphologically altered occlusion or by different jaw positions. ${ }^{38}$ Michelotti et al. (2006) examined adolescents with unilateral posterior crossbite and age-matched patients with normal occlusion, and they failed to detect differences in how the subjects distributed their body weight on their foot area, or in how quickly their bodies swayed. ${ }^{39}$ Furthermore, the unilateral posterior crossbite does not appear to be associated with leg length differences in young adolescents. ${ }^{40}$

A recent review suggests an association linking the cervical spine, the stomatognathic system, and craniofacial pain, but most of this information is inconclusive and comes from poor-quality studies, rated as levels $3 \mathrm{~b}, 4$, and 5 based on Sackett's classification. We believe that better designed studies are needed in order to clarify the actual influence that the cervical spine has on the stomatognathic system and craniofacial pain.

\section{CONNECTIONS BETWEEN THE TRIGEMINAL SYSTEM AND NERVOUS STRUCTURES IN- VOLVED IN MAINTAINING POSTURE}

Numerous anatomical connections have been described between trigeminal systems and the nervous structures involved in maintaining posture. The mesencephalic nucleus of the trigeminus (MNT), which extends itself from the dorsal portion of the spinal trigeminal nucleus to the caudal part of the superior colliculus, is a sensorial nucleus with unique characteristics. The cells of this nucleus are not central neurons, but protoneurons with the function of ganglionic cells. Kandel (1991) showed that this nucleus can be considered the equivalent of a sensitive peripheral ganglion. They are pseudounipolar neurons that send the axon externally to the NCS, while the other connections establish intra-axial contacts. ${ }^{1}$ This may explain the sensitivity of SS patients to different descendant stimuli (stress, anxiety, etc.) or ascendant stimuli (proprioceptive inputs from spine, feet, legs). ${ }^{42}$

In the MNT, neurons associated with extraocular muscles are present as along with the primary afferent neurons associated with muscles of MM, tooth pulp, and periodontal ligaments. ${ }^{43,44}$ From the MNT, the neural pathways connect with the cerebellum and with the reticular formation and the medial, inferior, lateral, and superior vestibular nuclei. ${ }^{44-46}$ They then continue to the spinal motor neurons and the extra-ocular efferents.

Indirect evidence suggests a functional connection between the vestibular and trigeminal systems. For example, painful trigeminal stimulation can trigger or modulate auditory and vestibular symptoms, such as spontaneous nystagmus, in patients with migraine headaches. ${ }^{48,49}$ Buisseret et al. (1990) used peroxidase injection into the oculomotor muscles as a marker to observe diffusion into several areas of the nervous system: the Gasser node, the interpolaris and the caudalis portion of the spinal trigeminal nucleus, the paratrigeminal nucleus, and the dorsal horn of the cervical spinal cord $(\mathrm{C} 1-\mathrm{C} 2)^{45}$

Studies have also revealed connections between the main nucleus of the trigeminus and the oral, interpolar and caudal portion of the spinal trigeminal nucleus on the one hand, with the vestibular nucleus and the prepositus nucleus of the hypoglossus on the other. ${ }^{46}$ The prepositus nucleus is part of a small group of nervous cells adjacent to, but not connected with, the nucleus of the hypoglossus. It is also an important nerve center for controlling the position and movement of the eyes, due to its strict relationship with the vestibular nuclei, the cerebellum, and the oculomotor nuclei. ${ }^{50}$

The MNT also makes connections with lobes IX and $\mathrm{X}$ of the cerebellum. ${ }^{44}$ Bilateral trigeminocollicular connections have been described in pigs, rats, and cats, where these connections involve the principal trigeminal nucleus and MNT. ${ }^{51-53}$ The superior colliculus is a relay center in the midbrain that receives visual, somesthetic, and proprioceptive afferent fibers; it is involved in postural, 
motor, and gait control, as well as gaze movements. In fact, the lateral SC region is part of the neuronal circuits that are involved in eye movements, and the region is associated with eyelid movements and lid saccades. This lateral superior colliculus region receives trigeminal somatic afferent information. ${ }^{52}$

Studies have shown the relationship between among dental occlusion, the oculomotor system, and visual stabilization. Evidence of a correlation between eye and dental occlusion came from the use of mandibular orthopedic repositioning appliances (MORAs), which simultaneously modify mandibular position and visual focusing tests using the Maddox rod and the Berens prismatic bars. These phenomena gradually disappear after removal of the appliance. $^{54}$

Associations also exist between TMD and oculomotor function. Recently, Monaco et al. $(2003,2004)$ suggested a much higher prevalence of ocular convergence defects in TMD adults presenting limited maximal opening, myofascial pain, and pain in the neck shoulder area, as well as in children with functional mandibular latero-deviation ${ }^{55,56}$ compared with healthy individual. In addition, TMD patients showed significantly lower binocular function, measured as convergence and positive fusional vergence, than did control healthy subjects. ${ }^{57}$ Connections were also observed with the cerebellum, and with many brainstem nuclei (nucleus of the solitary tract, dorsal reticular formation, cuneate nucleus), with the dorsal horn of the spinal cord (from C1-C5) and with the trigeminal primary afferent neurons. ${ }^{1,44-46,58,59}$

All of these anatomical connections suggest that portions of the trigeminal system strongly influence the coordination of posture and sight. It seems likely that sensory information from SS proprioceptive receptors is processed in tandem with information from the vestibular and oculomotor systems. Changes in trigeminal stimulations can cause an imbalance in the vestibular and oculomotor systems. ${ }^{15}$

Nociceptive inputs from TMJ, MM, posterior cervical muscles, and neck and shoulder muscles can produce a continuous afferent bombardment onto the spinal trigeminal nucleus. ${ }^{60}$ Such repeated nociceptive activation of the caudalis nucleus can produce central sensitization, which refers to increased excitability of brainstem nociceptive neurons. Two other types of stimulation leading to increased excitability of nociceptive neurons, individually or together, are temporal summation and activation of glial cells (microglia and astrocytes). ${ }^{61}$

The temporal summation is an increase in the intensity of pain experienced as a consequence of repeated stimulation. For example, efforts to maintain correct eye movement when the trigeminal nociceptive afferent stimulus is changing in intensity (trigeminotectal pathways) can lead to increased sensitivity (central fatigue), which causes progressive reduction in motoneuron recruitment and neurotransmitter synthesis, and concomitant loss of muscular force. Such central fatigue can be more severe in TMD patients than in healthy subjects, and it may explain the occurrence of ocular convergence defects. ${ }^{57}$ Moreover, if SS proprioceptive information is inaccurate, the control of the head position may be affected.

If confirmed by other studies, these hypotheses may explain why patients with dento-musculo-skeletal pathologies are predisposed to postural problems. By using stimuli of different intensity and duration, further studies should ascertain the threshold at which oculomotor and vestibular problems occur, and how the treatment of the TMDs affects posture.

\section{CONNECTIONS BETWEEN THE TRIGEMINAL SYSTEM AND MUSCLE-FASCIAL CHAINS}

Another basic element of the correlation between SS and human posture is the existence of muscle-fascial chains (MFC). Fasciae are dense, fibrous connective tissues that interpenetrate and surround the human body to protect, nourish and hold organs in place. ${ }^{62,63}$ Three layers of fasciae exist: superficial, deep and visceral. Deep fasciae surrounds muscles, bones, nerves and blood vessels and is densely populated with myofibroblasts and several types of receptors (nociceptors, proprioceptors, mechanoreceptors, chemoreceptors, thermoreceptors). Myofibroblasts are fascial cells that are created as a response to mechanical stress and actively contract in a smooth, muscle-like manner. ${ }^{64-66}$

The fascial system is important not only because it can passively distribute tension in the body muscles when mechanically stimulated, but also because it contains mechanoreceptors and possesses an autonomous contractile ability that influences the tension of the fasciae. The stimulation of intrafascial mechanoreceptors (mostly interstitial and Ruffini endings) causes the vegetative nervous system and the CNS to change the tension in intrafascial myofibroblasts and regulate fascial pre-tension. ${ }^{67}$ These tensions are transmitted along the MFC, thereby influencing the posture of the entire body.

An MFC is a group of muscles that are connected through the fasciae and are longitudinally positioned in the human body. They run in the same direction and overlap in a continuous chain, like tiles on a roof, which efficiently conducts tension. ${ }^{68,69}$ All of the muscles in the chain are mutually dependent and behave as if they were a single muscle. The existence of MFC may explain why disorders of the MM functions such as chewing and swallowing, can be transmitted to distal musculature. 
Cervical posture may also be influenced by stimuli from the lower limbs. Tecco et al. (2007) have compared cervical posture in lateral skull radiographs between healthy subjects and subjects who had anterior cruciate ligament injury of the left knee. They found that the latter group showed significant head extension compared to the healthy subjects. ${ }^{70}$ Dvorak and Dvorak (1990) injected a hydro-saline solution into the transverse processes of $\mathrm{C} 7$ and used electromyography to observe muscle contraction in zones distal from the spinal metamer where the injection was made. ${ }^{71}$ This seems to indicate that, because of the connections within the fascial system, change in any part of the body may create a disorder in another. For example, a contracted masseter muscle transmits its tension to homolateral SCM, and such connections may explain the influence of the SCM on mandibular movements. ${ }^{72}$ The MFC may also explain why an anterior cruciate ligament injury influences muscular electromyography activity of masseter, anterior temporalis, posterior cervicals, sternocleidomastoid, and upper and lower trapezius.

\section{CONCLUSION}

According to the literature reviewed, we believe that there are real correlations between posture and the SS. However, due to the complexity of the factors involved, existing studies have left important gaps in understanding. Therefore, further controlled investigations of the long-term clinical effects of different stomatognathic inputs are necessary.

Postural alterations may reflect a general lack of equilibrium in the individual. ${ }^{2}$ Consequently, the various signals that reach the CNS, and the importance that each signal is given, will no longer be perfectly balanced with the motor responses, and the motor responses may no longer be adequate. ${ }^{74}$ In this way, an increase in postural swaying may indicate a general malaise caused by problems in the SS. Our clinical experience suggests that an interdisciplinary approach is reliable and sufficient for making diagnoses and developing treatment plans. This approach should involve a variety of experts in postural rehabilitation, including physiatrists, orthopedists, psychologists, physical therapists, dentists, oculists, optometrists, and ear, nose, and throat specialists.

\section{REFERENCES}

1. Kandel ER, Schwartz JH, Jessell TM. Principles of Neural Science, 3rd ed. New York: Elsevier Science Publication Co; 1991.

2. Bolmont B, Gangloff P, Vouriot A, Perrin Ph. Mood states and anxiety influence abilities to maintain balance control in healthy human subjects. Neurosci Lett. 2002;329:96-100.

3. The Academy of Prosthodontics, Glossary of Prosthodontic Terms. J Prosthet Dent. 1999;81:101.

4. Nakahara H, Nakasato N, Kanno A, Murayama S, Hatanaka K, Itoh H, et al. Somatosensory-evoked fields for gingiva, lip, and tongue. J Dent Res. 2004;83:307-11.

5. Storm C, Wänman A. A two-year follow-up study of temporomandibular disorders in a female Sami population: validation of cases and controls as predicted by questionnaire. Acta Odontol Scand. 2007;65:341-7.

6. Gangloff P, Louis JP, Perrin PP. Dental occlusion modifies gaze and posture stabilization in human subjects, Neurosci Lett. 2000;293:203-6.

7. Hellsing E, McWilliam J, Reigo T, Spangfort E. The relationship between craniofacial morphology, head posture and spinal curvature in 8, 11 and 15 years old children. Eur J Orthod. 1987;9:254-64.

8. Huggare JA, Raustia AM. Head posture and cervicovertebral and craniofacial morphology in patients with craniomandibular dysfunction. Cranio. 1992;10:173-8.

9. Kritsineli M, Shim YS. Malocclusion, body posture, and temporomandibular disorder in children with primary and mixed dentition. J Clin Pediatr Dent. 1992;16:86-93.

10. Milani RS, De Periere DD, Lapeyre L, Pourreyron L. Relationship between dental occlusion and posture. Cranio. 2000;18:127-34.

11. Bracco P, Deregibus A, Piscetta R. Effects of different jaw relations on postural stability in human subjects, Neurosci Lett. 2004;356:228-30.

12. Fujimoto M, Hayakawa I, Hirano S, Watanabe I. Changes in gait stability induced by alteration of mandibular position. J Med Dent Sci. 2001,48:131-6.

13. Lund P, Nishiyama T, Moller E. Postural activity in the muscles of mastications with the subjects upright, inclined, and supine. Scand J Dent Res. 1970;78:417-24.

14. Tingey EMK, Buschang PH, Thorockmorton GS. Mandibular rest position:a reliable position influenced by head support and body posture. Am J Orthod Dentofacial Orthop. 2001;120:614-22.

15. Gangloff P, Perrin PP. Unilateral anaesthesia modifies postural control in human subjects. Neurosci Lett 2002;330:179-82.

16. Sforza C, Tartaglia GM, Solimene U, Morgun V, Kaspranskiy RR, Ferrario VF. Occlusion, sternocleidomastoid muscle activity, and body sway: a pilot study in male astronauts. Cranio. 2006;24:43-9.

17. Nobili A, Adversi R. Relationship between posture and occlusion: a clinical and experimental investigation. Cranio. 1996;14:274-85.

18. Lippold C, Danesh G, Schilgen M, Derup B Hackenberg L. Relationship between thoracic, lordotic, and pelvic inclination and craniofacial morphology in adults. Angle Orthod. 2006;76:779-85.

19. Huggare J, Pirttiniemi P, Serlo W. Head posture and dentofacial morphology in subjects treated for scoliosis. Proc Finn Dent Soc. 1991;87:151-8.

20. Lippold C, Van den Bos L, Hohoff A, Danesh G, Ehmer U. Interdisciplinary study of orthopedic and orthodontic findings in preschool infants. J Orofac Orthop. 2003;64:330-40.

21. Ben-Bassat Y, Yitschaky M, Kaplan L, Brind I. Occlusal patterns in patients with idiopathic scoliosis. Am J Orthod Dentofacial Orthop. 2006;130:629-33.

22. Yoshino G, Higashi K, Nakamura T. Changes in weight distribution at the feet due to occlusal supporting zone loss during clenching. Cranio. 2003;21:271-8.

23. Ishijima T, Hirai T, Koshino H, Konishi Y, YokoyamaY. The relationship between occlusal support and physical exercise ability. J Oral Rehabil. 1998;25:468-71.

24. Chakfa AM, Mehta NR, Forgione AG, Al-Badawi EA, Lobo SL, Zawawi KH. The effect of stepwise increases in vertical dimension of occlusion on isometric strength of cervical flexors and deltoid muscles in nonsymptomatic females. Cranio. 2002;20:264-73.

25. Ishii H. A study on the relationships between imbalance of stomatognathic function and asymmetry of craniofacial morphology, and the center of gravity of the upright posture. Osaka Daigaku Shigaku Zasshi 1990;35:517-56. 
26. Hackney J, Bade D, Clawson A. Relationship between forward head posture and diagnosed internal derangement of the temporomandibular joint. J Orofacial Pain. 1993;7:386-90.

27. Olmos SR, Kritz-Silverstein D, Halligan W, Silverstein ST. The effect of condyle fossa relationships on head posture. Cranio. 2005;23:48-52.

28. Simons DG, Travell J, Simons LS. Myofascial pain and dysfunction: the trigger point manual. Vol 1. 2nd ed. Baltimore: Williams \&Wilkins; 1999.

29. Fernández-de-las-Peñas C, Alonso-Blanco C, Cuadrado ML, Gerwin RD, Pareja JA. Trigger points in the suboccipital muscles and forward head posture in tension-type headache. Headache. 2006;46:454-60.

30. Gresham H, Smithells PA. Cervical and mandibular posture. Dental Rec. 1954;74:261-4.

31. Ayub E, Glasheen-Wray M, Krauss S. Head posture: a case report of the effects on the rest position of the mandible. J Orthoapaedic and Sports Physical Therapy. 1984;5:179-60.

32. Friedman MH, Wusberg I. Screening procedures for temporomandibular joint dysfunction. Am Fam Phys 1982;25:157-60.

33. Janda V. Some aspects of extracranial causes of facial pain. J Prosthet Dent. 1981;56:484-7.

34. Mannheimer JS, Rosenthal RM. Acute and chronic postural abnormalities as relatedto craniofacial pain and temporomandibular disorders. Dent Clin North Am .1991;35:185-209.

35. D'Attilio M, Epifania E, Ciuffolo F, Salini V, Filippi MR, Dolci M, et al. Cervical lordosis angle measured on lateral cephalograms; findings in skeletal class II female subjects with and without TMD: a cross sectional study. Cranio. 2004;22:27-44.

36. Gangloff P, Louis JP, Perrin PP. Dental occlusion modifies gaze and posture stabilization in human subjects, Neurosci Lett. 2000;293:203-6.

37. Ferrario VF, Sforza C, Schmitz JH, Taroni A. Occlusion and centre of foot pressure variation:is there a relationship? J Prosthet Dent. 1996;76:302-8

38. Ferrario VF, Sforza C, Serrao G, Fragnito N, Grassi G. The influence of different jaw positions on the endurance and electromyographic pattern of the biceps brachii muscle in young adults with different occlusal characteristics. J Oral Rehabil. 2001;28:732-9.

39. Michelotti A, Buonocore G, Farella M, Pellegrino G, Piergentili C, Altobelli S, et al. Postural stability and unilateral crossbite: is there a relationship? Neurosci Lett. 2006;392:1404.

40. Michelotti A, Farella M, Buonocore G, Pellegrino G, Piergentili C, Martina R. Is unilateral posterior crossbite associated with leg length inequality? Eur J Orthod. 2007;29:622-6.

41. Armijo Olivo S, Magee DJ, Parfitt M, Major P, Thie NM. The association between the cervical spine, the stomatognathic system, and craniofacial pain: a critical review. J Orofac Pain. 2006;20:271-87.

42. Meerssman GM, Esposito GM. Valutazione delle relazioni fra occlusione e postura. Il Dentista moderno. 1988;6:5-9.

43. Dessem D, Taylor A. Morphology of jaw-muscle spindle afferents in the rat. J Comp Neurol. 1989;282:389-403.

44. Pinganaud G, Bourcier F, Buisseret-Delmas C, Buisseret P. Primary trigeminal afferents to the vestibular nuclei in the rat: existence of a collateral projection to the vestibulo-cerebellum. Neurosci Lett. 1999;264:133-6.

45. Buisseret-Delmas C, Buisseret P. Central projections of extraocular muscle afferents in cat. Neurosci Lett. 1990;109:48-53.

46. Buisseret-Delmas C, Compoint C, Delfini C, Buisseret P. Organisation of reciprocal connections between trigeminal and vestibular nuclei in the rat. J Comp Neurol. 1999;409:153-68.

47. Lee WY, Okeson JP, Lindroth J. The relationship between forward head posture and temporomandibular disorders. J Orofac. Pain 1995;9:161-7.

48. Marano E, Marcelli V, Di Stasio E, Bonuso S, Vacca G, Manganelli F, et al. Trigeminal Stimulation Elicits a Peripheral Vestibular Imbalance in Migraine Patients. Headache. 2005;45:325-31

49. Vass Z, Shore SE, Nuttall AL, Miller JM. Direct evidence of trigeminal innervation of the cochlear blood vessels. Neuroscience. 1998;84:559-67.

50. Williams PL, Warwick R. Gray's Anatomy, 36th ed. Philadelphia Saunders: Williams \& Warwick eds 1980.

51. Ndiaye A, Pinganauda G, Frans VanderWerfb F, Buisseret-Delmas C, Buisseret P. Connections between the trigeminal mesencephalic nucleus and the superior colliculus in the rat. Neurosci Lett . 2000;294:17-20.

52. Dauvergne C, Ndiaye A, Buisseret-Delmas C, Buisseret P, VanderWerf F, Pinganaud G. Projections from the superior colliculus to the trigeminal system and facial nucleus in the rat. J Comp Neurol. 2004;478:233-47.

53. Huerta MF, Frankfurter AJ, Harting JK. The trigeminocollicular projection in the cat: patch-like endings within the intermediate gray. Brain Res. 1981 27;211:1-13.

54. Milani RS, de Periere DD, Micallef JP. Relationship between dental occlusion and visual focusing. J Craniomandibular Pract. 1998;16:109-18.

55. Monaco A, Streni O, Marci MC, Sabetti L, Giannoni M. Convergence defects in patients with temporomandibular disorders. J Craniomandibular Pract. 2003;21:190-5.

56. Monaco A, Streni O, Marci MC, Sabetti L, Marzo G, Giannoni M Relationship between mandibular deviation and ocular convergence. J Clin Pediatr Dent. 2004;28:135-8.

57. Cuccia AM, Caradonna C. Binocular motility system and temporomandibular joint internal derangement: a study in adults. Am J Orthod Dentofacial Orthop. 2008;133:640.e15-20.

58. Pompeiarto M, Palacios JM, Mengodb G. Distribution and Cellular Localization of mRNA Coding for SHT Receptor in the Rat Brain: Correlation with Receptor Binding. Neurosci Lett. 1992;12:440-53.

59. Marfurt CF, Rajchert DM. Trigeminal primary afferent projections to "non-trigeminal" areas of the rat central nervous system. J Comp Neurol. 1991;303:489-511.

60. Fernández-de-las-Peñas C, Cuadrado ML, Pareja JA. Myofascial trigger points, neck mobility and forward head posture in unilateral migraine. Cephalalgia. 2006;26:1061-70.

61. Xie XF, Zhang S, Chiang CY, Hu JW, Dostrovsky JO, Sessle BJ. Involvement of glia in central sensitization in trigeminal subnucleus caudalis (medullary dorsal horn). Brain Behav Immun. 2007;21:63441.

62. Schleip R, Klingler W, Lehmann-Horn F. Active fascial contractility: Fascia may be able to contract in a smooth muscle-like manner and thereby influence musculoskeletal dynamics. Med Hypotheses. 2005;65:273-7.

63. Paoletti, Serge. The Fasciae: Anatomy, Dysfunction \& Treatment. Seattle, WA: Eastland Press, 2006.

64. Schleip R, Klingler W, Lehmann-Horn F. Active fascial contractility: Fascia may be able to contract in a smooth muscle-like manner and thereby influence musculoskeletal dynamics". Med Hypotheses. 2005;65:273-7.

65. Tomasek J, Gabbiani G, Hinz B, Chaponnier C, Brown R. Myofibroblasts and Mechanoregulation of Connective Tissue Remodelling. Molecular Cell Biology. 2002;3:350-2.

66. Schleip R. Fascial plasticity - A new neurobiological explanation: Part 2. Journal of Bodywork and Movement Therapies 2003;7:11-9.

67. Myers TW. (2002). Anatomy trains: myofascial meridians for manual and movement therapists. Edinburgh: Churchill Livingstone, 2001.

68. Valentino B, Fabozzo A, Melito F. The functional relationship between the occlusal plane and the plantar arches. An electromyography study. Surg Radiol Anat. 1991;13:171-4.

69. Valentino B, Maccauro L, Mango G, Melito F, Fabozzo A. Electromyography for the investigation and early diagnosis of scoliosis. Anat Clin. 1985;7:55-9

70. Tecco S, Colucci C, Caraffa A, Salini V, Festa F. Cervical lordosis in patients who underwent anterior cruciate ligament injury: a crosssectional study. Cranio. 2007;25:42-9.

71. Dvorak J, Dvorak V. Manual medicine: diagnostics. 2nd ed, New York: Thieme Medical Publishers; 1990.

72. Shimazaki K, Matsubara N, Hisano M, Soma K. Functional Relationships Between the Masseter and Sternocleidomastoid Muscle Activities During Gum Chewing:The Effect of Experimental Muscle Fatigue. Angle Orthod. 2006;76:452-8.

73. Tecco S, Salini V, Tetè S, Festa F. Effects of anterior cruciate ligament (ACL) injury on muscle activity of head, neck and trunk muscles: a cross-sectional evaluation. Cranio. 2007;25:177-85.

74. Sforza C, Ferrario VF. Anatomical bases of body posture: methods and applications. Mondo Ortod. 2008:1;25-32. 